\title{
Exploring design cognition in voice-driven sound sketching and synthesis
}

\author{
Stefano Delle Monache ${ }^{10000-0003-2210-8410]}$ and Davide \\ Rocchesso $^{2[0000-0002-0849-7766]}$ \\ 1 Faculty of Industrial Design Engineering, Delft University of Technology, the \\ Netherlands stefano.dellemonache@gmail.com \\ 2 Dept. of Mathematics and Computer Science, University of Palermo, Italy \\ davide.rocchesso@unipa.it
}

\begin{abstract}
Conceptual design and communication of sonic ideas are critical, and still unresolved aspects of current sound design practices, especially when teamwork is involved. Design cognition studies in the visual domain represent a valuable resource to look at, to better comprehend the reasoning of designers when they approach a sound-based project. A design exercise involving a team of professional sound designers is analyzed, and discussed in the framework of the Function-BehaviorStructure ontology of design. The use of embodied sound representations of concepts fosters team-building and a more effective communication, in terms of shared mental models.
\end{abstract}

Keywords: Sound design · Collaboration · Design cognition.

\section{Introduction}

We witness an essential process of convergence of inquiries in sound design towards the broader field of design research. From different angles, sound studies, sonic interaction design (SID), computer science, auditory cognition studies, and sonification research are challenging the inherent cooperative and collaborative, yet ambiguous nature of listening and hearing, as method and means to contribute to better everyday environments for the living [2|43]. More recently, sound design research has been unfolding its interest in the interaction- and information-centered use of sound in computational artefacts, towards the study of the process of designing sound. There are a variety of reasons to study sound designing. Researchers may want to have an understanding of the actual activities carried out by practitioners and their status [52. Others may investigate the design process with the goal of improving the practice [12]16. Other loci of interest may inquiry designing sound with the aim of developing appropriate design tools and supporting technologies throughout the various stages of the process [7]14. Finally, other research approaches, whether bottom-up (e.g., case studies and design explorations [32 44]) or top-down (e.g., reference frameworks and systems [50|4]), may wish to achieve and provide a more general and 
abstracted explanation of thinking patterns in sound design tasks, the design process and methods that practitioners may look at.

Indeed, one main problem that sound practitioners strive to deal with is the communication and evaluation of a design, that is sound, in which the distance between the intermediary representations and the final product is perceived as very short. The intrinsic ambiguity of sound and listening do affect both the collaboration between peers and the contact points with stakeholder in general [10, p.35]: Communicating and elaborating concepts through sound can be hazardous, especially in the early interactions with clients; design solutions on sound are difficult to argue, especially when designers overindulge in the description of the sound-producing mechanisms rather than accounting for the global sensory experience; as a consequence, the evaluation of sound design proposals often takes the prosaic form of the individual preference of the client, whenever it is not based on psychoacoustic metrics for sound quality assessment [36 40.

In the practice, sound creation and production rather unfold, within the overall design process, as an individual activity kept separate and asynchronous from the global product development, the effect of which undermines the participation and communication with stakeholders, both horizontally and vertically, and brings about a tendency to anticipate, early in the process, the creation of assortments of selected variations of highly refined sounds [46 47].

Since the $2^{\text {nd }}$ International Symposium "Les Journées du Design Sonore" $\sqrt{3}$ in 2004, a growing corpus of additive knowledge on sound design has been produced (see 24/39/49/18/37/3/43/17, for a comprehensive overview). Such body of knowledge outlines a landscape of descriptive models of designing sound, whose central proposal is a closed loop of sound evaluation and design that advances through rapid prototyping and iterative improvement: Research through design workshops, design critique and explorations, and controlled experiments with sonic interactive artifacts and tools are primarily focused on the design activity in the conceptual and embodiment stages of the design process.

It turns out, however, that little is known about how sound designers think, generate and develop ideas. Given their multidisciplinary background [38, how do sound designers approach projects? For example, do they favor a search process in the problem space, like industrial designers do, or in the solution space, like engineers apparently prefer [28]

Designing (sound) takes place in people's minds. Despite the market availability of countless types of computational tools for sound analysis and production, the very first creative act happens in the designer's mind. Understanding sound design thinking becomes crucial to create the next generation of design tools, computational or not, to aid the generation and communication of auditory concepts. In this respect, sound design research may look at design cognition studies in the visual domain, as reference framework of relevant topics, and rigorous and

\footnotetext{
3 The symposium, organized by Frédérique Guyot (LAPS-design) and Patrick Susini (Ircam) in collaboration with the French Acoustical Society, took place at Centre Pompidou, Paris, France, in 2004 (https://www.centrepompidou.fr/cpv/resource/ cazjxnn/rLLRyR).
} 
formal methods and ontologies to investigate individual and collaborative design dynamics involving sound [1125].

In this area of study, protocol analysis is the established, empirical method, commonly used to inquiry well-defined design phenomena, such as novice-expert differences in problem structuring and organization of cognitive actions, the effect of the "structeredness" of ideation methods on cognition, the role of design representations and sketching, the conditions for design fixation and its effect on the novelty of ideas, and in general the cognitive processes involved in design moves [15. Typically, audiovideo documentations of design sessions are transcribed and parsed in segments, that is the smallest units of analysis that can be time-based, reflect turn-taking (e.g., in team dynamics) or other rationale (e.g., decision-making), according the granularity and the objective of the study. Segments are coded according to meaningful schemes that may well-represent the particulars of the case in question. Finally, various kinds of qualitative and quantitative analyses can be carried out in order to derive an understanding of the design phenomena under scrutiny [23[29].

In this work, we analyze the protocol of a design session involving a team of professional sound designers engaged in vocal sketching the sound of two car models (e.g., idle engine, driving, braking), with the aid of a computational tool for voice-driven sound synthesis [14. We apply the Function-Behavior-Structure (FBS) ontology of design [29, chap. 13] as coding scheme, from which we derive information on the team dynamics and productivity, the role-taking, the designing style and process unfolding. The FBS ontology is a formal coding scheme which takes in account the cognitive processes emerging as transitions in the design space, and precisely in terms of transformations between classes of issues which are intrinsic to any design domain: i) the purpose of the artifact, that is the Function; its imagined performance, that is the Behavior Expected; iii) its form and configuration, that is the Structure; iv) the resulting performance, that is the Behavior derived from the Structure (see further, Section 3).

The paper is organized as follows: the next Section provides an overview of topics on cognition in conceptual design activity which can be relevant to investigate in design teamwork involving sound; Section 3 introduces the sound design session, and the coding procedure using the FBS ontology of design; we analyze and discuss the session in Section 4

\section{Conceptual design cognition at a glance}

It has been argued that the next generation of CAD systems will be defined by four main characteristics, and namely cognition, collaboration, concepts, and creativity [20]. The majority of the research in design cognition concerns the disciplines of architectural design, engineering design, and product design. The research revolves around the two main paradigms of design as search in the problem space [21] or design as exploration and co-evolution [22 48, and focuses on the processes of information gathering and structuring, the role of long-term memory, schema activation in working memory, semantic processing, mental syn- 
thesis and sketch-based reasoning (see 25|26 for a systematic review of protocol studies on conceptual design cognition). These processes are essentially inspected from a visuo-spatial perspective, if one excludes the role of the phonological loop for verbal design information [428].

Such a structured knowledge and methodological approach are still missing in the realm of aural collaborative creativity and design. Certainly, auditory imagery and cognition represent a vast field of research which received an increasing attention in the past several years. The majority of the studies, originating from experimental psychology and neuroscience, are aimed at the understanding of the human abilities to generate and manipulate mental auditory images, where music and language are the preferred foci of interest: For instance, it has been shown that auditory images contain both depictive and descriptive components, that is some relationships of the auditory stimuli are preserved (e.g., pitch and temporal properties), while others rather "sound like" (e.g., loudness), and that the reinterpretation (i.e., figure-ground segregation) of a given stream of sounds is more difficult in auditory imagery than in auditory perception, especially when subvocalization is blocked [27. Functional MRI studies showed that conceptual acoustic processing, that is thinking about a sound even when implicitly presented through visual words, involves a partial reinstatement of the brain activity during the perceptual experience of the same acoustic features 31.

Empirical frameworks on the role of the active body as inherent mediator between perception and the cognitive processing of music (and sound) have been proposed, wherein the sonic experience emerges in interaction, as complex network of intentional states and internal models of observable patterns, that are acquired through knowledge and skills [35. Within this framework, recent researches not only showed that vocal imitations of nonverbal sounds encode salient acoustic features into some other vocal features [33, while gestural metaphors are exploited to illustrate auditory sensations and causal representations of sonic concepts [34, but also explored their use as cognitive devices to enable and support sketch-based reasoning in conceptual sound design [13].

Embodied cognition, concepts, and creativity are at the center of frameworks for designing the next generation of sonic information and interactions 43 45, where experimental applications of body-centered auditory display and sonification are finding their way in walking interactions in mixed reality, physical rehabilitation and motor learning, sensory alteration and emotional design [51]. On the other side, process-based studies on sound design are still embryonic [11, if one excludes the existing literature on creative thinking in music processes: The ill-defined problem of composing a piece of music is solved through iterative, non-linear stages of insight (i.e., musical inspiration), problem restructuring and proliferation 9. Cognitive processes in music composition can reflect an analytic, horizontal approach to the sequential writing of the musical parts, or develop vertically, that is implying a strong conceptualization phase of the whole in advance, before the actual production: The "sound designer" style has been characterized by an in-depth, horizontal exploration and original use of tools, 
where iterations are especially concentrated in the re-execution and revision of sound segments in the recording phases [1].

Horizontal and vertical compositional strategies strongly resonate with the dual mechanism model of design problem-solving: Lateral transformations of an idea are divergent and associative, are facilitated by ill-structured representations (i.e., conceptual sketches), and widen the problem space, while vertical transformations are convergent and inferential, are facilitated by well-structured representations (e.g., prescriptive sketches and blueprints), and deepen the problem space [21. It has been shown how sketch-based reasoning facilitates the reorganization and creation of new knowledge [5]. It can be argued that the main criticality of sound design is the unbalanced use of well-structured representations [12, due to the lack of proper tools that afford sketching in the established workflow, at least in the acceptation widespread in the visual domain [10, p.35].

Cooperation and collaboration in conceptual sound design are not common practices, where design in a natural setting, e.g. in a design firm, is typically carried out by teams with multidisciplinary background. The study of design teams may reveal several insights on design thinking. One main advantage of examining design teams is that think-aloud protocols are naturally enforced and concurrent with the execution of the task. It has been shown that early collaborations are the most effective and improve consistency in mental models, and yet that design teamwork do not necessarily brings about a higher productivity, compared to the individual activity: Apparently, the experienced individual designer is equipped with all the necessary expertise to act as a unitary system (i.e., a team, in which the expertise are allocated by role-taking instead), whereby the semantic coherence of the team composition affects positively the quality of the final designs [15].

Design ontologies have been proposed to formalize and analize the design process. The Function-Behavior-Structure (FBS) coding framework was used to observe an industry team (a business consultant, three mechanical engineers, an electronic business consultant, an ergonomicist, and an industrial design student), involved in a brainstorming session: The distribution of word count and turns variation throughout the design episodes provided coarse quantitative observations on the quality of the team interaction, in terms of producing a shared mental model, whereby the analysis of transitions between FBS design issues produced fine-grained representations of the design process at the individual and team level [30].

The same coding scheme was applied to measure the designing styles of teams of industrial design students and mechanical engineering design students, that is observing whether the designers' focus on the problem space or on the solution space may be specific to design disciplines, and how it may affect the team building and composition [28]: The Problem-Solution Index (PSI) was proposed as ratio measurement, computed over the total occurrences of design issues representing problem formulations (i.e., the function and the expected behavior) and design issues representing solutions to the formulated problem (i.e., the structure, and the behavior derived from the structure). The same measurement was 


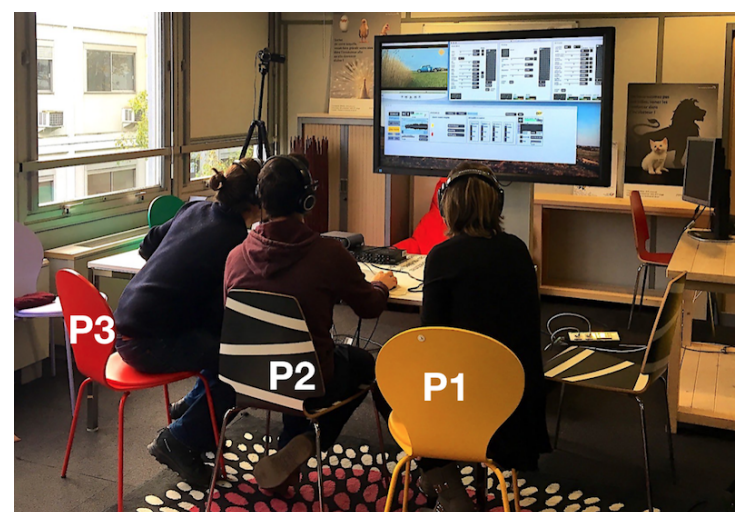

Fig. 1: Team composition: project manager (P1), audio engineer (P2), sound designer (P3).

applied to investigate how the structuredness of concept generation techniques affects the cognitive focus of teams towards the problem space or the solution space in the early stage of the design process [19.

Taken together, this concise survey of relevant studies on team design cognition prompts a path of open questions in the domain of aural creativity and design. While the discipline of sonic interaction design has been proposing and accumulating a variety of methods, frameworks and techniques [4]18 13 166], to our knowledge no formal and structured inquiries have been carried out yet, in terms of their impact on sound design cognition. In the next Section we describe the sound design task, along with a brief, operational discussion of the FBS ontology, the coding procedure and the conventions.

\section{Collaborative sketching with voice-driven sound synthesis}

The vocal sketching exercise took place during a sound design workshop organized in collaboration with the audio research team of a vehicle manufacturing company. The team, including two sound designers, two audio engineers, and one project manager, was split in two groups in order to derive two protocols that could be analyzed and compared. The fictitious task was to re-design the EV (i.e., Electric Vehicle) engine sound version of two combustion engine car models. The assignment was delivered as follows:

Theme - A brand-new voice for an old car: How would the Citröen 21DS Rally and the Peugeot 205 GTI sound today if they were electric?

Objective: Re-think and design their sound according to the current brand values, optimistic, smart, human.

Time constraints: The design task duration is 90 minutes, split in 2 sessions of 45 minutes per car model. 


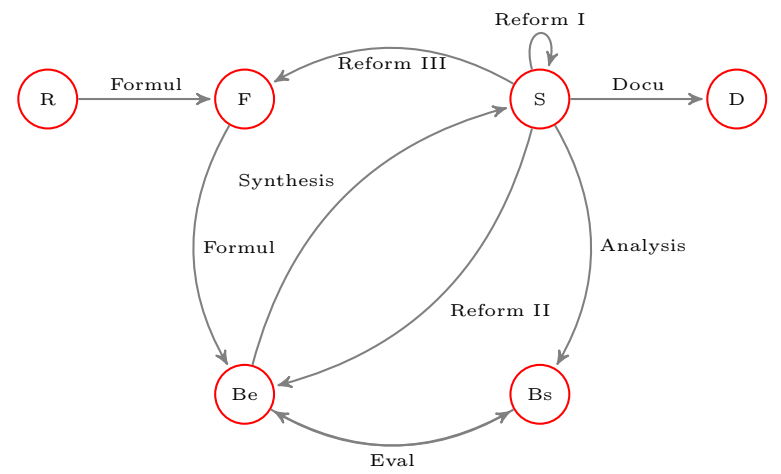

Fig. 2: The FBS framework. The codes represent the issues. Arcs are labeled according to the design processes.

The teams were provided with a computational tool for voice-driven sound synthesis, to support their sketching activity: The tool affords the externalization of synthetic sound impressions by means of mixtures of sound models that can be set, played and shared as instances of vocal utterances. We refer to our previous work for the in-depth description and evaluation of the too 14 . The groups were also provided with silenced videos of the two car models, in order to video-prototype their design. The teams tackled the Citröen sound first, and the Peugeot sound design later. All the design tasks were video-recorded. In this paper, we report the protocol analysis of one team composed by one sound designer, one audio engineer and the project manager, shown in Figure 1

\subsection{The Function-Behavior-Structure coding procedure}

According to the FBS framework, any design artifact can be described and modeled as a set of semantic transitions, that is cognitive processes, between three classes of ontological variables, and namely the purpose of the artifact, its imagined and emerging performances, the components of the artifact and their compositional relationships. As shown in Figure 2, the FBS coding scheme maps these three classes on six design issues, which are represented as codes: The final goal of any designing activity is to transform a set of requirements $(\mathrm{R})$ and functions (F) into a set of design descriptions of the artifact at hand (D).

In the practice, the transcript of the design session is parsed in design moves, that is segments of utterances, gestures, and any other kinds of representation, which are labeled according to the design issues. Design moves-issues are linked according to causal transformations. Typically, the designer formulates the function $(\mathrm{F})$, based on the requirements $(\mathrm{R} \rightarrow \mathrm{F})$, which are usually derived from the brief. The expected behavior $(\mathrm{Be})$ reflects the performance imagined to fulfill the function $(\mathrm{F} \rightarrow \mathrm{Be})$. Hence, the designer synthesizes the imagined performance in

\footnotetext{
4 The tool in action: https://vimeo.com/271826511.
} 


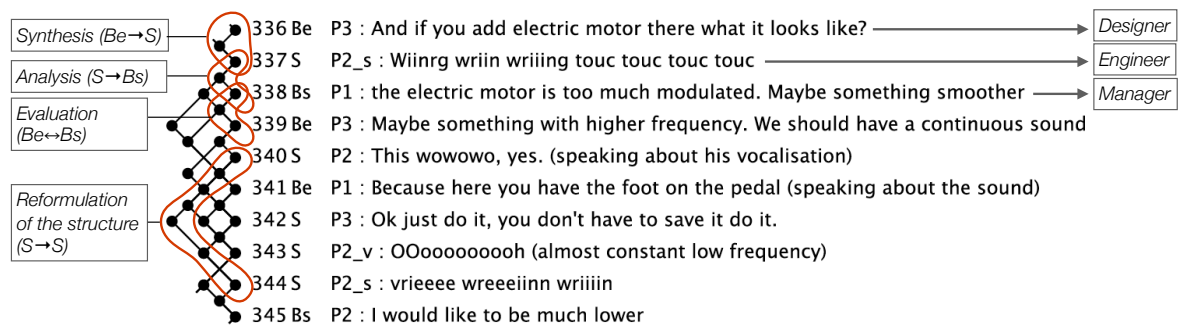

Fig. 3: Linkograph of an excerpt of 12 FBS-coded moves, extracted from the Citröen protocol in Figure 4a. Links and nodes are visible, and examples of transitions between issues are highlighted: synthesis (e.g., $336 \rightarrow 337$ ), reformulation of the structure (e.g., $340 \rightarrow 344$ ), analysis (e.g, $337 \rightarrow 338$ ) and evaluation (e.g, 338 $\rightarrow 339)$.

externalized representations, that is structural configurations of elements and formal relationships $(\mathrm{Be} \rightarrow \mathrm{S})$. The inspection of the structure can lead to three types of reformulations: the revision of the structure itself (Reformulation I, $\mathrm{S} \rightarrow \mathrm{Bs}$ ); the different articulation of the expected performance (Reformulation II, $\mathrm{S} \rightarrow \mathrm{Be}$ ); the proper reformulation of the function by lateral thinking the actual sketch or prototype (Reformulation III, $\mathrm{S} \rightarrow \mathrm{F}$ ). The analysis transition occurs once the structure is produced $(\mathrm{S} \rightarrow \mathrm{Bs})$, and the actual performance based on the structure is assessed with respect to the performance expected (evaluation, $\mathrm{Be} \leftrightarrow \mathrm{Bs}$ ). Eventually, this finite-state loop of design processes among design issues leads to the documentation of external design descriptions $(\mathrm{S} \rightarrow \mathrm{D})$.

In coding the transcript of the two design sessions (i.e., the Citröen 21DS rally and the Peugeot $205 \mathrm{GTI}$ ), we followed a set of conventions, extensively reported in [11, that we introduced to fully capture the sound designers' intentions, as they find themselves involved in discussing by means of verbalizations, vocalizations, iconic gestures accompanying the utterances, and synthesized sounds driven by both the vocal control and the mouse interaction on the graphic user interface of the sketching tool.

Once the coding procedure is completed, the emerging network of design moves can be organized and represented as a linkograph, where the relations among nodes, such as the density, the distance between links, the amount of links, their direction, distribution and patterns, are used to inspect the productivity and the behavior of the designer(s) involved in the actual process 23. Figure 3 shows the linkographic representation of 12 moves extracted from the protocol of the Citröen sound design session. The linkograph is generated by feeding the software LiNKODER ${ }^{5}$ with the FBS-coded protocol [41]. In the next Section, we analyze and compare the two episodes, in order to derive a global picture of the team cooperation and dynamics.

${ }^{5}$ LiNKODER, a design protocol analysis tool: https://sites.google.com/a/linkoder. com/linkoder/home 


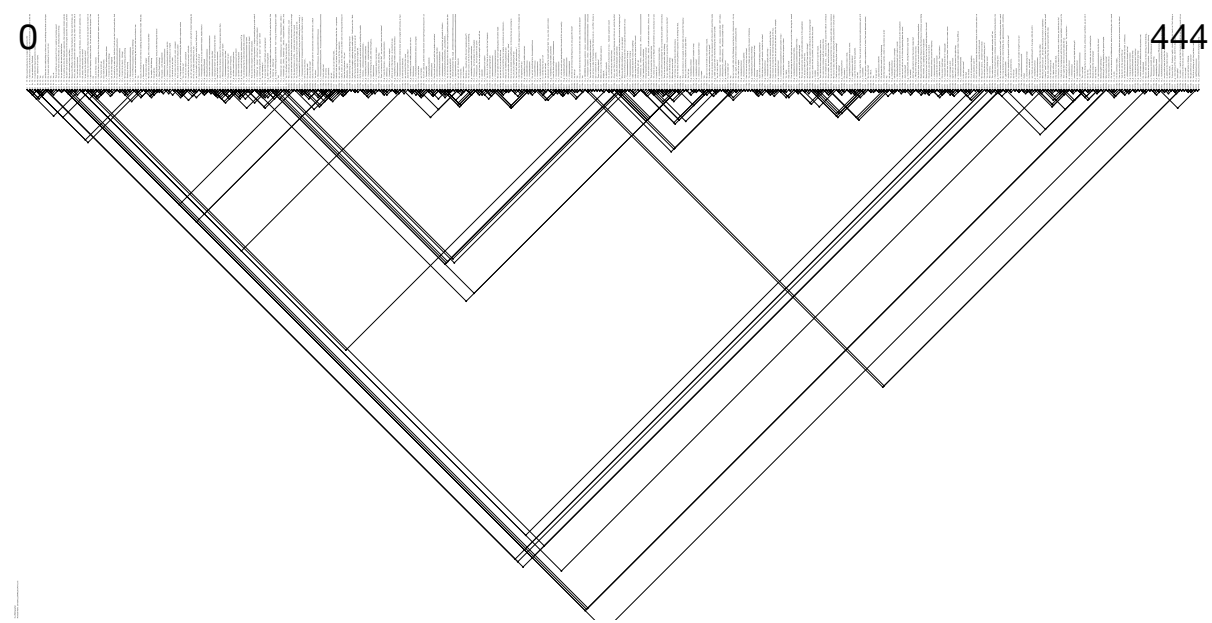

(a) Linkograph of the Citröen sound design session, computed over 444 segments, FBScoded protocol.

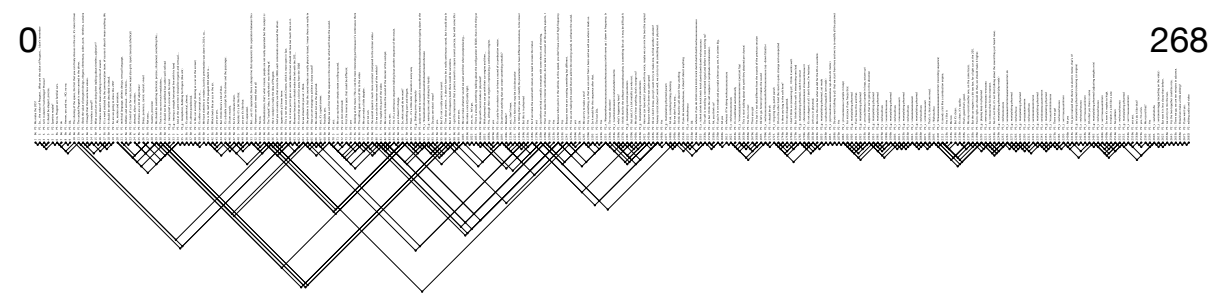

(b) Linkograph of the Peugeot sound design session, computed over 268 segments, FBS-coded protocol.

Fig. 4: Linkographic representations of the two sound design episodes, as generated by the software LiNKODER 41.

\section{Analysis and discussion}

The Citröen sound session (E1) resulted in a protocol of 444 moves, whereas 268 segments were retained for coding the Peugeot sound session (E2). From the visual inspection of their linkographic representations, shown respectively in Figures $4 \mathrm{a}$ and $4 \mathrm{~b}$, we can notice that E1 is characterized by a longer link span (mean 10.5, STD 45.5), which denotes either a longer incubation of ideas, where sketching serves as external memory function, or a typical team behavior where members may relate to their previous moves, regardless of the other moves intervened in the meanwhile. E2 linkograph is much more cohesive, with a shorter link span $($ mean $=5.3, \mathrm{STD}=10.3)$, which suggests a rather unitary behavior of team. The two different team behaviors, that is the approach to the design task, do not necessarily imply a higher or lower productivity. The link index is 


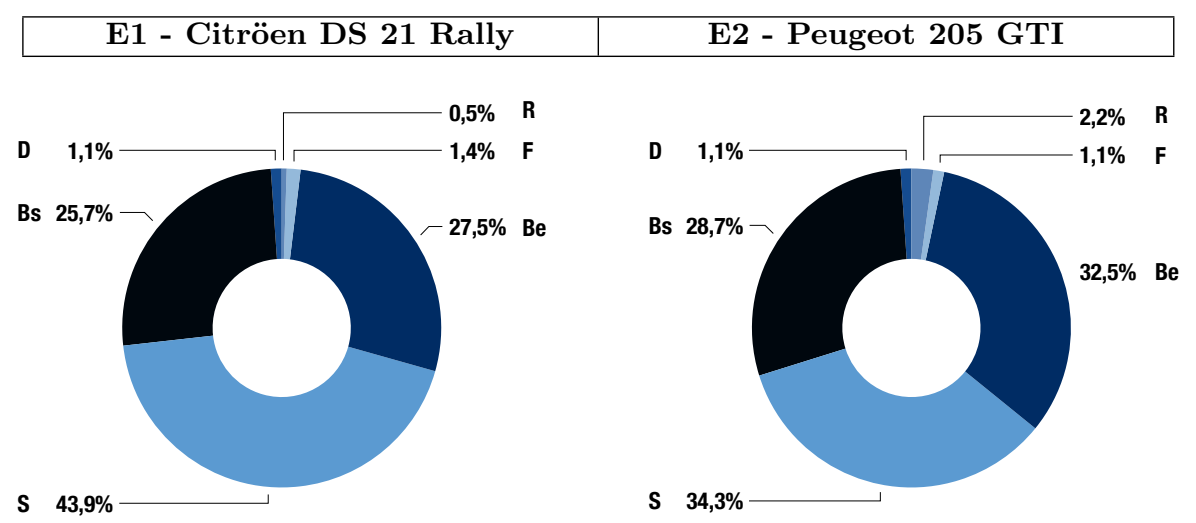

(a) Distribution of issues. The structural issues (S, Bs) represent more than the $60 \%$ of all the codes.

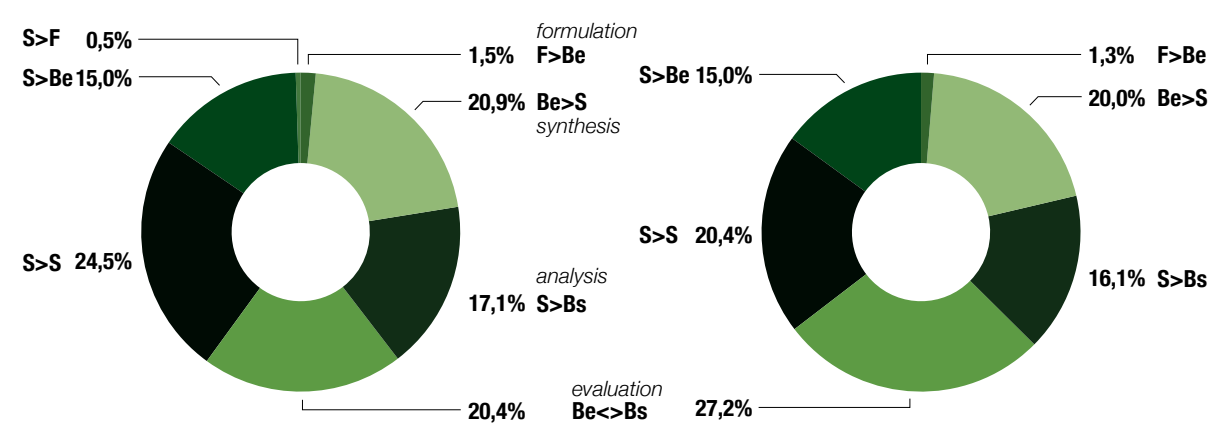

(b) Distribution of the processes. The team efforts are especially focused on producing and evaluating the sound $(\mathrm{S} \rightarrow \mathrm{S} ; \mathrm{Be} \leftrightarrow \mathrm{Bs})$.

Fig. 5: Distribution of issues and processes in the two design sessions (Citröen on the left, Peugeot on the right).

a coarse indicator of the productivity, based on the ratio between the number of links and the number of moves, and represents the amount of linking activity in terms of effort to achieve a synthesis. In the case of E1 and E2 the link indexes are essentially comparable $(\mathrm{E} 1, \mathrm{~L} . \mathrm{I} .=2.04$; E2, L.I. $=1.97)$. The different shapes of the linkographs rather suggest a different organization of the teamwork.

The distribution of issues and processes in E1 and E2, shown in Figure 5a and 5b, allows a closer inspection of the team behavior. The Mann-Whitney non-parametric test of both distributions does not show a statistical significance (issues, Mann-Whitney, $U=12, P>.05$; processes, Mann-Whitney, $U=17$, $P>.05)$, hence hinting at a similar approach of the team towards the task solution, in the two sessions. Indeed, the percentage of D issues is identical and yet the low percentage can be mainly attributed to the fact that only shared and 


\begin{tabular}{|c|c|}
\hline \multicolumn{2}{|c|}{ Problem-Solution Index } \\
\hline \hline E1 & 0.42 \\
\hline E2 & 0.56 \\
\hline
\end{tabular}

Table 1: The designing approach of the team was rather focused on the solution space (PSI < 1.). The PSI is the ratio between the design issues representing the problem formulation $(\mathrm{F}, \mathrm{Be})$ and the design issues concerned with the problem solution (S, Bs).

\begin{tabular}{|c|c|c||c|c||c|c||c|c|}
\hline & \multicolumn{2}{|c|}{ Verbalizations } & \multicolumn{2}{c|}{ Vocalizations } & \multicolumn{2}{c|}{ Gestures } & \multicolumn{2}{c|}{ Synth Snd } \\
\hline \hline & E1 & E2 & E1 & E2 & E1 & E2 & E1 & E2 \\
\hline P1 & 67 & 58 & 0 & 0 & 0 & 0 & 0 & 0 \\
\hline P2 & 77 & 54 & 19 & 1 & 28 & 4 & 32 & 2 \\
\hline P3 & 141 & 87 & 34 & 19 & 18 & 22 & 28 & 21 \\
\hline Tot & 285 & 199 & 53 & 20 & 46 & 26 & 60 & 23 \\
\% & $(64,2)$ & $(74,2)$ & $(11,9)$ & $(7,5)$ & $(10,4)$ & $(9,7)$ & $(13,5)$ & $(8,6)$ \\
\hline
\end{tabular}

Table 2: Team activity: Occurrence of types of expressions in the two design sessions $\mathrm{E} 1$ and $\mathrm{E} 2$ (P1 = manager; P2 = engineer; P3 = designer).

\begin{tabular}{|c|c|c||c|c||c|c||c|c|}
\hline & \multicolumn{2}{|c||}{$\begin{array}{c}\text { P1 - } \\
\text { Manager }\end{array}$} & \multicolumn{2}{c||}{$\begin{array}{c}\text { - P2 - } \\
\text { Engineer }\end{array}$} & \multicolumn{2}{c||}{$\begin{array}{c}\text { - P3 - } \\
\text { Designer }\end{array}$} & \multicolumn{2}{c|}{$\begin{array}{c}\text { Team } \\
\text { (Tot) }\end{array}$} \\
\hline \hline & E1 & E2 & E1 & E2 & E1 & E2 & E1 & E2 \\
\hline$<\mathbf{C M}^{\mathbf{4}}$ & 1 & 3 & 8 & 7 & 18 & 14 & 27 & 24 \\
\hline $\left.\mathbf{C M}^{\mathbf{4}}\right\rangle$ & 3 & 8 & 29 & 8 & 26 & 15 & 58 & 31 \\
\hline$\left\langle\mathbf{C M}^{\mathbf{4}}\right\rangle$ & 1 & 1 & 5 & 2 & 3 & 8 & 9 & 11 \\
\hline
\end{tabular}

Table 3: Backward, forward and bidirectional critical moves in the two design sessions E1 and E2. The superscript indicates the criticality threshold.

agreed-upon documentations were coded. Taken together, the two episodes reveal a design activity especially centered on structural issues ( $\mathrm{S}, \mathrm{Bs},>60 \%)$, where the cognitive efforts are mainly allocated to the reformulation of the structure and the evaluation of the resulting sound design.

In the practice, the two linkographic representations depict a shift in the effectiveness of the team's communication and collaboration. In E1, the team members rather cooperate, take their roles, explore and understand the vocal sketching tool. The intense work on the structural issues can be ascribed to the novelty of using the vocalizations as sketching means, and to the difficulty to obtain from the tool synthetic representations coherent with their intentions. This led the team to reconsider often the expected performance, that is the imagined sound ( $\mathrm{S} \rightarrow \mathrm{Be}$, Reformulation II). In E2, the slightly lower percentage of structural issues and reformulations suggests that the team made sense of their collaboration through the sketching tool, and achieved the same results 


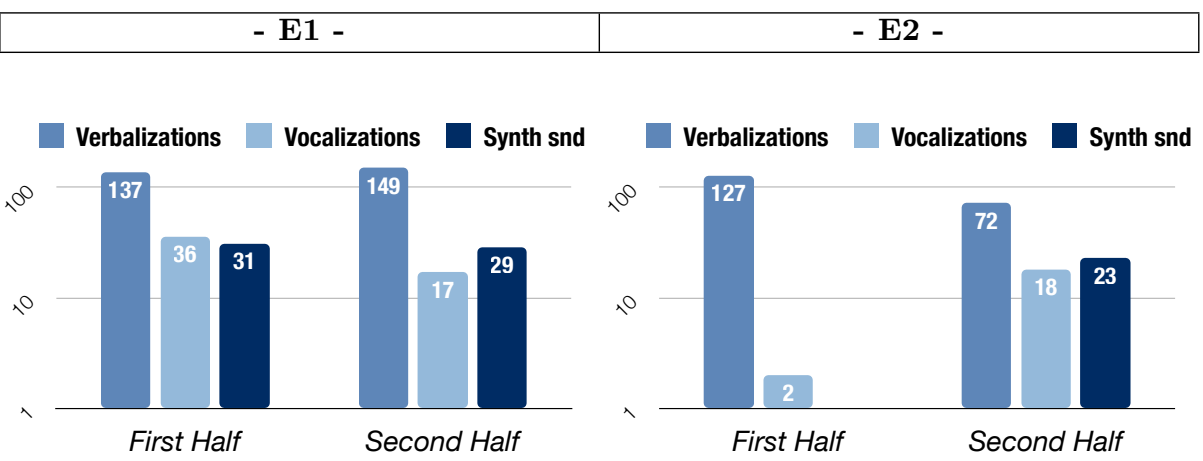

Fig. 6: Verbal and nonverbal expressions in the two design sessions.

(i.e., D issues) in little more than half the moves of E1, though in the same amount of time (i.e., 45 minutes).

The team mindset is mainly addressed to the actual sound production, rather than its conceptualization (i.e., $\mathrm{F} \rightarrow \mathrm{Be}, \mathrm{Be} \rightarrow \mathrm{S}$ ). More in detail, table 1 reports the problem-solution index value for $\mathrm{E} 1$ and $\mathrm{E} 2$, which reflects the attitude of the team towards the design process, whether focused on the conceptualization of the design problem (P-S I. $>1$.) or of the design solution (P-S I. $<1$.). The shift to the diverse approaches may depend not only on the background, but also on the specific design task [28].

Table 2 reports the group activity in terms of use of verbalization, vocalizations and gestures, and externalized synthesized sounds. As expected, verbalthinking is the main channel of communication. The different amount of use of other forms of communication by the team reflects the role and background of the members. The sound designer (P3) was apparently the most active in both episodes, where the audio engineer (P2) became less engaged in using vocalizations and gestures in E2. The project manager (P1) only interacted by talking.

However, the quality of the individual contribution in the two episodes is reported in table 3 . The critical moves (CMs) are moves with a high number of links, based on a significant threshold typically set in order to obtain around $10-12 \%$ CMs from the total number of moves [23, p.73]. The links between the critical moves are arranged in nodes of reasoning which may lead forward, thus denoting acts of synthesis $(\mathrm{CM}>)$, or backward, thus representing acts of evaluation $(<\mathrm{CM})$. Bidirectional moves $(<\mathrm{CM}>)$ are associated to rapid shift of divergent and convergent thinking. Critical moves represent turning points in the unfolding of the design process. Whereas P2 and P3 were the main drivers of the process in E1, the team found a stronger integration and produced a more balanced process in E2.

In this respect, the count of the types of expressions in the two halves of the protocols of the two sessions is shown in Figure 6. Although the total percentage of verbalizations is even increasing in E2 (see Table 2, last row, second column), the two halves in E2 are very different in nature, whereby the drop 
of verbalizations and increase of nonverbal representations in the second half stresses the achievement of shared mental models, as a consequence of a better integrated conceptualization phase in the first half of the session (see also the peculiar shape of the corresponding linkograph in Figure 4b). To conclude, the design exercise acted throughout the two design sessions as an effective teambuilding tool, fostering more effective communication and collaboration between members with multidisciplinary background.

\section{Conclusions}

Designing is a process of construction of representations, from early, unstructured ideas of products, systems, services, etc. held in the mind towards the final artefacts. We showed how a cognition-based inquiry of sound design can reveal several aspects of team dynamics in conceptual design activities, which are considered critical and still unresolved in the current practices.

In the post-workshop comments, the participants reported a major frustration caused by several limitations of the computational sketching tool, that is first and foremost a lack of immediacy of use of the user interface and of technical integration in their established workflow (i.e., DAWs), whereby the voice-driven sound synthesis approach prompted clear expectations on the creation and shaping of the sound sketches. Nonetheless, the participants remarked the high value of collaboration experienced, by sketching through voice-based representations. They reported that for the first time, at least in their everyday workflow, they experienced to work collaboratively around a project. They especially remarked how through collaboration diverse approaches and ideas emerged. They found the overall workshop useful for team-building and reflecting on the role of creativity in their everyday sound design practice.

Protocol studies and ontologies of design, such as the FBS framework, represent valuable resources to investigate the process of sound design. In this respect, the establishment of design cognition studies in the sound design domain provides a novel, yet complementary perspective on the study of embodied sound cognition.

Understanding how representations of sound designs are externalized for communication and collective transformation purposes becomes crucial to open sound design practices to truly participatory approaches, when users and stakeholders are involved not only as subjects, but especially as partners.

\section{References}

1. Barbot, B., Webster, P.R.: Creative thinking in music. In: Lubart, T. (ed.) The Creative Process: Perspectives from Multiple Domains, pp. 255-273. Palgrave Macmillan UK, London (2018)

2. Barney, A., Voegelin, S.: Collaboration and consensus in listening. Leonardo Music Journal 28, 82-87 (2018) 
3. Barrass, S.: Sonic information design. Journal of Sonic Studies (2018), Special Issue on Sonic Information Design, https://www.researchcatalogue.net/view/558606/558686

4. Brazil, E.: A review of methods and frameworks for sonic interaction design: Exploring existing approaches. In: Ystad, S., Aramaki, M., Kronland-Martinet, R., Jensen, K. (eds.) Auditory Display - 6th International Symposium, CMMR/ICAD 2009, Copenhagen, Denmark, May 18-22, 2009, Revised Papers, Lecture Notes in Computer Science, vol. 5954, pp. 41-67. Springer (2010)

5. Brun, J., Le Masson, P., Weil, B.: Designing with sketches: the generative effects of knowledge preordering. Design Science 2, e13 (2016)

6. Caramiaux, B., Altavilla, A., Pobiner, S.G., Tanaka, A.: Form follows sound: Designing interactions from sonic memories. In: Proceedings of the 33rd Annual ACM Conference on Human Factors in Computing Systems. pp. 3943-3952. CHI '15, ACM, New York, NY, USA (2015)

7. Carron, M., Rotureau, T., Dubois, F., Misdariis, N., Susini, P.: Speaking about sounds: a tool for communication on sound features. Journal of Design Research 15(2), 85-109 (2017)

8. Cash, P., Maier, A.: Prototyping with your hands: the many roles of gesture in the communication of design concepts. Journal of Engineering Design 27(1-3), 118-145 (2016)

9. Collins, D.: A synthesis process model of creative thinking in music composition. Psychology of music 33(2), 193-216 (2005)

10. Delle Monache, S., Baldan, S., Boussard, P., Del Piccolo, A., Dendievel, C., Lemaitre, G., Lachambre, H., Houix, O., Rocchesso, D.: Interactive prototypes realized with the SkAT-VG tool. Deliverable, SkAT-VG project (2017)

11. Delle Monache, S., Rocchesso, D.: Cooperative sound design: A protocol analysis. In: Proceedings of the Audio Mostly 2016. pp. 154-161. AM '16, ACM, New York, NY, USA (2016)

12. Delle Monache, S., Rocchesso, D.: To embody or not to embody: A sound design dilemma. In: Fontana, F., Gulli, A. (eds.) Machine sounds, Sound machines - Proc. of the XXII CIM Colloquium on Music Informatics. pp. 93-100 (November 2018)

13. Delle Monache, S., Rocchesso, D.: Sketching sonic interactions. In: Filimowicz, M. (ed.) Foundations in Sound Design for Embedded Media, A Multidisciplinary Approach, pp. 79-101. Routledge, New York (2019)

14. Delle Monache, S., Rocchesso, D., Bevilacqua, F., Lemaitre, G., Baldan, S., Cera, A.: Embodied sound design. International Journal of Human-Computer Studies 118, $47-59(2018)$

15. Dinar, M., Shah, J.J., Cagan, J., Leifer, L., Linsey, J., Smith, S.M., Hernandez, N.V.: Empirical studies of designer thinking: past, present, and future. Journal of Mechanical Design 137(2), 021101-0211013 (2015)

16. Erkut, C., Serafin, S., Hoby, M., Sårde, J.: Product sound design: Form, function, and experience. In: Proceedings of the Audio Mostly 2015 on Interaction With Sound. pp. 10:1-10:6. AM '15, ACM, New York, NY, USA (2015)

17. Filimowicz, M.: Foundations in Sound Design for Embedded Media, A Multidisciplinary Approach. Routledge, London, UK (2019)

18. Franinović, K., Serafin, S.: Sonic interaction design. Mit Press (2013)

19. Gero, J.S., Jiang, H., Williams, C.B.: Design cognition differences when using unstructured, partially structured, and structured concept generation creativity techniques. International Journal of Design Creativity and Innovation 1(4), 196-214 (2013) 
20. Goel, A.K., Vattam, S., Wiltgen, B., Helms, M.: Cognitive, collaborative, conceptual and creative-four characteristics of the next generation of knowledge-based cad systems: a study in biologically inspired design. Computer-Aided Design 44(10), 879-900 (2012)

21. Goel, V.: Creative brains: designing in the real world. Frontiers in Human Neuroscience 8, 241 (2014)

22. Goldschmidt, G.: The backtalk of self-generated sketches. Design Issues 19(1), 72-88 (2003)

23. Goldschmidt, G.: Linkography: unfolding the design process. MIT Press (2014)

24. Grimshaw, M.: Game Sound Technology and Player Interaction: Concepts and Developments. IGI global (2010)

25. Hay, L., Duffy, A.H.B., McTeague, C., Pidgeon, L.M., Vuletic, T., Grealy, M.: A systematic review of protocol studies on conceptual design cognition: Design as search and exploration. Design Science 3, e10 (2017)

26. Hay, L., Duffy, A.H.B., McTeague, C., Pidgeon, L.M., Vuletic, T., Grealy, M.: Towards a shared ontology: A generic classification of cognitive processes in conceptual design. Design Science 3, e7 (2017)

27. Hubbard, T.L.: Auditory imagery: empirical findings. Psychological bulletin 136(2), $302(2010)$

28. Jiang, H., Gero, J.S., Yen, C.C.: Exploring designing styles using a problemsolution division. In: Design Computing and Cognition'12, pp. 79-94. Springer (2014)

29. Kan, J.W., Gero, J.S.: Quantitative methods for studying design protocols. Springer (2017)

30. Kan, J.W., Gero, J.S., Tang, H.H.: Measuring cognitive design activity changes during an industry team brainstorming session. In: Design Computing and Cognition'10, pp. 621-640. Springer (2011)

31. Kiefer, M., Sim, E.J., Herrnberger, B., Grothe, J., Hoenig, K.: The sound of concepts: Four markers for a link between auditory and conceptual brain systems. Journal of Neuroscience 28(47), 12224-12230 (2008)

32. Lemaitre, G., Houix, O., Visell, Y., Franinović, K., Misdariis, N., Susini, P.: Toward the design and evaluation of continuous sound in tangible interfaces: The spinotron. International Journal of Human-Computer Studies 67(11), 976-993 (2009)

33. Lemaitre, G., Jabbari, A., Houix, O., Misdariis, N., Susini, P.: Vocal imitations of basic auditory features. The Journal of the Acoustical Society of America 139(1), 290-300 (2016)

34. Lemaitre, G., Scurto, H., Françoise, J., Bevilacqua, F., Houix, O., Susini, P.: Rising tones and rustling noises: Metaphors in gestural depictions of sounds. PloS one 12(7), e0181786 (2017)

35. Leman, M., Maes, P.J., Nijs, L., Van Dyck, E.: What is embodied music cognition? In: Bader, R. (ed.) Springer Handbook of Systematic Musicology, pp. 747-760. Springer (2018)

36. Lyon, R.H.: Product sound quality-from perception to design. Sound and vibration 37(3), 18-23 (2003)

37. Meelberg, V., Özcan, E.: Editorial: Designing our sonic lives. Journal of Sonic Studies (6) (2018), Special Issue on Sound Design, https://www.researchcatalogue.net/view/239747/239748/0/0

38. Özcan, E., van Egmond, R.: Product sound design: An inter-disciplinary approach? In: Undisciplined! Design Research Society Conference (2009), http://shura.shu.ac. $\mathrm{uk} / 531 /$ 
39. Pauletto, S.: Perspectives on sound design. The New Soundtrack 4(2), v-vi (2014)

40. Pedersen, T.H., Zacharov, N.: How many psycho-acoustic attributes are needed. Journal of the Acoustical Society of America 123(5), 3163-3163 (2008)

41. Pourmohamadi, M., Gero, J.S.: LINKOgrapher: An analysis tool to study design protocols based on FBS coding scheme. In: Culley, S., Hicks, B., McAloone, T., Howard, T., Clarkson, J. (eds.) ICED 2011 Proc. of the 18th International Conference on Engineering Design, Impacting Society through Engineering Design. vol. 2: Design Theory and Research Methodology, pp. 294-303 (2011)

42. Purcell, A., Gero, J.S.: Drawings and the design process: A review of protocol studies in design and other disciplines and related research in cognitive psychology. Design studies 19(4), 389-430 (1998)

43. Rocchesso, D., Delle Monache, S., Barrass, S.: Interaction by ear. International Journal of Human-Computer Studies (2019), accepted for publication

44. Rocchesso, D., Polotti, P., Delle Monache, S.: Designing continuous sonic interaction. International Journal of Design 3(3), 13-25 (December 2009)

45. Roddy, S., Bridges, B.: Sound, ecological affordances and embodied mappings in auditory display. In: Filimowicz, M., Tzankova, V. (eds.) New Directions in Third Wave Human-Computer Interaction: Volume 2 - Methodologies, pp. 231258. Springer International Publishing, Cham (2018)

46. Sander, H.: Listen! Improving the cooperation between game designers and audio designers. In: DiGRA '11 - Proceedings of the 2011 DiGRA International Conference: Think Design Play. DiGRA/Utrecht School of the Arts (January 2011)

47. Sanz Segura, R., Manchado Pérez, E.: Product sound design as a valuable tool in the product development process. Ergonomics in Design 26(4), 20-24 (2018)

48. Schon, D.A.: The reflective practitioner: How professionals think in action. Basic Books (1984)

49. Serafin, S., Franinović, K., Hermann, T., Lemaitre, G., Rinott, M., Rocchesso, D.: Sonic interaction design. In: Hermann, T., Hunt, A., Neuhoff, J.G. (eds.) The Sonification Handbook, chap. 5, pp. 87-110. Logos Publishing House, Berlin, Germany (2011)

50. Susini, P., Houix, O., Misdariis, N.: Sound design: an applied, experimental framework to study the perception of everyday sounds. The New Soundtrack 4(2), 103$121(2014)$

51. Tajadura-Jiménez, A., Väljamäe, A., Bevilacqua, F., Bianchi-Berthouze, N.: Bodycentered auditory feedback. In: Norman, K.L., Kirakowski, J. (eds.) The Wiley Handbook of Human Computer Interaction, pp. 371-403. John Wiley \& Sons (2017)

52. Zattra, L., Misdariis, N., Pecquet, F., Donin, N., Fierro, D.: Analysis of sound design practices [asdp]. research methodology. In: Fontana, F., Gulli, A. (eds.) Machine sounds, Sound machines - Proc. of the XXII CIM Colloquium on Music Informatics. pp. 168-175 (November 2018) 